Published in final edited form as:

Schizophr Res. 2013 November ; 150(0): 498-504. doi:10.1016/j.schres.2013.08.038.

\title{
Theory of Mind and Social Judgments in People at Clinical High Risk of Psychosis
}

\author{
Kristin Healeya ${ }^{a}$, David L. Penn ${ }^{a},{ }^{*}$, Diana Perkins ${ }^{b}$, Scott W. Woods ${ }^{c}$, and Jean Addington ${ }^{d}$ \\ aDepartment of Psychology, University of North Carolina at Chapel Hill, Chapel Hill NC, United \\ States \\ ${ }^{b}$ Department of Psychiatry, University of North Carolina at Chapel Hill, Chapel Hill NC, United \\ States \\ 'Department of Psychiatry, Yale University, New Haven, CT, USA \\ dDepartment of Psychiatry, Faculty of Medicine, University of Calgary, Calgary AB, Canada
}

\begin{abstract}
Background-Social cognitive deficits are consistently reported in psychotic populations. Few studies have longitudinally investigated social cognition in clinical high-risk (CHR) populations.

Aims-Longitudinally examine theory of mind (ToM) and social judgments in a CHR sample to investigate stability of performance over time and potential ability to predict conversion to psychosis.

Method-147 CHR individuals and 85 help seeking controls (HSC) were assessed for up to 2 years; 28 participants developed psychosis across both groups. Generalized linear mixed models for repeated measures was used to examine change over time for ratings on the three social cognitive indices of ToM, trustworthiness, and approachability. Hierarchical regression was used to test whether social cognitive variables explain more variance in conversion than IQ.
\end{abstract}

Results-CHR individuals showed a positive bias in approachability judgments over time compared to HSC. Baseline ToM performance significantly $(\mathrm{p}<.05)$ predicted later conversion beyond IQ scores. These results were attenuated when controlling for baseline symptom level.

\footnotetext{
(C) 2013 Elsevier B.V. All rights reserved.

*Corresponding author. University of North Carolina at Chapel Hill, Department of Psychology, 250 Davie Hall, Chapel Hill NC 27599-3270. Tel: (919) 8435262.

Publisher's Disclaimer: This is a PDF file of an unedited manuscript that has been accepted for publication. As a service to our customers we are providing this early version of the manuscript. The manuscript will undergo copyediting, typesetting, and review of the resulting proof before it is published in its final citable form. Please note that during the production process errors may be discovered which could affect the content, and all legal disclaimers that apply to the journal pertain.

Declaration of Interest: None.

Conflict of Interest

All authors declare that they have no conflicts of interest.

Contributors

Author 1 (K. Healey), completed all statistical analyses and contributed substantially to all drafts of the manuscript. Author 2 (D.

Penn) aided in study design, data collection, and contributed substantially to all drafts of the manuscript. Authors 3 (D. Perkins) and 4

(S. Woods) aided in study design, data collection, and obtained funding. Author 5 (J. Addington) aided in study design, data collection, obtained funding, and contributed substantially to all drafts of the manuscript. All authors have approved the final manuscript.
} 
Conclusions-Although ToM deficits might predate conversion to psychosis; one must consider initial symptoms as well. Social judgments were not associated with conversion to schizophrenia.

\section{Keywords}

clinical high risk; schizophrenia; social cognition; theory of mind; social judgments; longitudinal study

\section{Introduction}

Similar to preventative work targeting medical illness, many research groups have focused on improving models of risk prediction in schizophrenia (Cannon et al., 2008). A central goal of this work is to enhance understanding of factors related to conversion to psychosis and illness progression. Preliminary evidence suggests that social cognition (SC) may be a viable target, as deficits in $\mathrm{SC}$ are present both during the active illness phase and in populations at clinical high risk (CHR) for developing psychosis (Green et al., 2008; Thompson et al., 2011). Specifically, there is some support for deficits in emotion recognition and theory of mind (ToM) prior to illness onset. Thus, the assessment of SC deficits in individuals at clinical high risk (CHR) of developing schizophrenia warrants further exploration, as it may lead to the identification of a putative risk marker. The present study examines the SC domains of ToM and social judgments in a CHR population.

Individuals with first episode and chronic schizophrenia show consistent impairments in theory of mind (ToM) (Sprong et al., 2007). ToM typically refers to the ability to infer others' mental states (Green et al., 2008). In CHR individuals, there is mixed support for deficits in ToM. These studies are largely cross-sectional in design and administer a heterogeneous group of ToM tasks. Couture et al. (2008) found no significant differences in higher order ToM performance (Eyes Task) between CHR and healthy control (HC) groups. Stanford et al. (2011) also found no significant differences between CHR and HC groups in Eyes Task performance, nor with other first-and second-order ToM tasks (False Belief, Strange Story Tasks). Effect sizes (cohen's d) computed from mean differences between HC and CHR groups were small in both studies $(d=.06-.35)$ (Cohen, 1988). Conversely, Chung et al. (2008) utilized higher order, verbal tasks of ToM (False Belief and Strange Story Task) and found significant differences between CHR individuals and HCs matched on age and IQ. Recent studies provide further evidence of significant deficits in higher order ToM performance in CHR groups, although they were unable to draw conclusions about deficits' association with later conversion (Green et al., 2012; Hur et al., 2013; Thompson et al., 2012).

There is one prior study in CHR individuals that assessed ToM longitudinally to predict progression to psychosis. Kim at al. (2011) examined performance in four lower and higher order ToM tasks in 49 ultra-high risk (UHR) individuals over time (mean=2.8 years). They found significant differences between converters $(n=13)$ and non-converters $(n=36)$ at baseline in both higher order verbal (False Belief task; $d=.74$ ) and lower order non-verbal (Cartoon Task; $d=.59$ ) tasks. Further, there was not a significant relationship between ToM and symptoms, consistent with previous work (Couture et al. 2008; Stanford et al., 2011). Findings support the predictive value of ToM deficits in later conversion to psychosis. Taken together, this suggests that due to inconsistent findings and cross sectional design, further longitudinal investigation of ToM deficits in clinical high-risk groups is warranted.

There is a less extensive literature investigating social judgments in psychotic spectrum illnesses. Social judgments include trustworthiness and approachability ratings, which 
require usage of nuanced, subtle information conveyed in facial appearances. Social judgments require integration of various sources of information, such as facial expression and previous experiences with similar looking individuals (Couture et al., 2008). Such evaluations are antecedents in decision-making processes that directly influence social behaviors. In chronic schizophrenia, findings are mixed regarding social judgments assessed with the trustworthiness task (Adolphs, 1998), although individuals with non-paranoid schizophrenia tend to rate unfamiliar faces as more trustworthy and with greater variability than controls (Baas et al., 2008a; Baas et al., 2008b; Couture et al., 2010; Haut and MacDonald, 2010; Pinkham et al., 2008). Comparatively little work has focused on social judgments in CHR populations. Baas et al. (2008b) found that individuals at genetic risk of developing psychosis (first degree family members) rated faces significantly trustworthier than healthy controls. Similarly, Couture et al. (2008) found that CHR individuals judged a subset of untrustworthy faces as significantly trustworthier than healthy controls. Positive biases in social judgments are consistent with a body of established social cognitive deficits in schizophrenia, whereby patients have difficulty recognizing and applying appropriate social information (Baas et al., 2008b).

The aim of the present study is to longitudinally investigate ToM and social judgment performance in a group of CHR individuals compared to a group of help seeking control (HSC) individuals. Here, we assess whether ToM and social judgment performance (a) differentially changes over time as a function of risk status and (b) predicts conversion to psychosis at baseline.

\section{Methods}

\subsection{Sample}

The sample consisted of 147 participants ( 85 males, 62 females) at CHR of developing psychosis with a mean age of $19.8(\mathrm{SD}=4.7)$ and 85 help-seeking control (HSC) participants (44 males, 41 females) with a mean age of $19.4(\mathrm{SD}=4.1)$ years. All data was collected as a part of the PREDICT study conducted at the Universities of North Carolina (59 CHR, 21 HS), Toronto (60 CHR, $40 \mathrm{HS}$ ), and Yale (28 CHR, $24 \mathrm{HS}$ ). The methods are described in detail in Addington et al. (Addington et al., 2012). All CHR participants met Criteria of Prodromal Syndromes (COPS) derived from the Structured Interview for Prodromal Syndromes (SIPS: McGlashan et al., 2010). A majority of CHR participants (N=145) met criteria for attenuated positive syndrome (APSS). Only three participants met criteria for genetic risk and deterioration (GRD), which requires either an affected first degree relative or the subject having schizotypal personality disorder (SPD) and $>30 \%$ drop in functioning on the General Assessment of Functioning (GAF) scale in the past 12 months.

The help-seeking control group (HSC) was comprised of individuals who had (1) responded to CHR recruitment and (2) presented with prodromal symptoms at phone screen but upon administration of the full interview did not meet prodromal criteria. The HSC group contains the following subgroups: (1) family high risk but no deterioration in GAF (n=16) (2) longstanding attenuated symptoms present for $>1$ year $(n=39)$ (3) current prodromal symptoms but symptoms were clearly due to another disorder $(n=2)(4)$ had only negative symptoms $(n=4)$ and $(5)$ symptoms that did not meet severity or frequency criterion $(n=24)$. HSC individuals were included as a clinically relevant control group that provide a more stringent test of conversion, as CHR and HSC individuals are more symptomatically similar to one another than to healthy controls.

The Structured Clinical Interview for DSM-IV (SCID-I: First et al., 1995) was administered to determine the presence of any axis I disorders. Exclusion criterion were: presence of an axis I psychotic disorder, IQ less than 70, or presence of a clinically significant CNS 
disorder that may be related to CHR symptoms. Individuals were also excluded for history of antipsychotic treatment, as the PREDICT study aimed to examine predictors of conversion to psychosis without the confound of antipsychotics. After conducting comprehensive clinical assessments to determine inclusion, participants completed the ToM and social judgment tasks. Additionally, the number of subjects at follow-up is inconsistent, thus missing subjects are accounted for by (1) dropping out of the study, (2) missing the assessment, and (3) conversion to psychosis (Table 1).

\subsection{Measures}

2.2.1 Clinical Measures-Prodromal syndrome and conversion criteria were assessed using the SIPS (McGlashan et al., 2010). Conversion meant that at least one of the five attenuated positive symptoms reached a psychotic level of intensity (rated 6) for a frequency of $\geq 1$ hour/day for 4 days/week during the past month or that symptoms seriously impacting functioning (e.g. severely disorganised or dangerous to self or others) (McGlashan et al., 2010). Symptoms were assessed with the Scale of Prodromal Symptoms (SOPS), which is comprised of 19 items in 4 symptom domains: positive, negative, general, and disorganized.

2.2.2 Theory of Mind Task-Theory of mind was assessed with the "Reading the Mind in the Eyes" task (Eyes Task: Baron-Cohen et al., 2001), a multiple choice task requiring the participant to infer mental/emotional states from 37 cropped photos of pairs of eyes.

Participants are instructed to select one of four words that best describe what the person is thinking or feeling based on their eyes. They are provided standardized definitions of word choices at their request. The Eyes Task was selected for its frequent usage in schizophrenia spectrum disorders (Pinkham et al., 2013).

2.2.3 Social Judgment Task-Social judgment was assessed with the Abbreviated Trustworthiness Task (Adolphs et al., 1998), in which participants viewed 42 unfamiliar faces and were asked to judge how much they would trust the pictured individual with their money or their life. They were also asked to judge how approachable these individuals appeared if they were to ask them for directions. They separately rated each face's trustworthiness and approachability on a 7 point scale ranging from -3 (very untrustworthy/ unapproachable) to +3 (very trustworthy/approachable). The images were chosen from a larger set of stimuli that was rated by 47 healthy subjects (Adolphs et al., 1998). The 42 images were selected to yield a wide range of trustworthy and approachable faces (22 untrustworthy, 20 trustworthy; 24 unapproachable; 18 approachable). None of these tasks were timed. The Trustworthiness Task was selected for its frequent usage in schizophrenia spectrum disorders (Pinkham et al., 2013).

2.2.4 Cognitive measures-IQ was assessed using the Wechsler Adult Intelligence Scale (WAIS-III: Wechsler, 1997) for participants age 16 and older (Arithmetic, Vocabulary, Information, Digit Span, and Block Design). The Wechsler Intelligence Scale for Children-Third Edition (WISC-III: Wechsler, 1991) was used for patients under 16 years of age (verbal and performance IQ). The Full Scale Intelligence Quotient (FSIQ) score was utilized as an indication of current cognitive function.

\subsection{Procedures}

The PREDICT study was a longitudinal study of predictors of conversion to psychosis. All raters were experienced research clinicians who demonstrated adequate reliability through administration of routine reliability checks. Gold standard post-training agreements on the discrimination between high risk (5) and psychotic (6) levels of intensity on the positive symptom items (the critical threshold for determining both initial eligibility and subsequent conversion status) were excellent (kappa $=0.90)$. The DSM-IV diagnoses were established 
with the SCID-I. Interrater reliability was determined at the start of the study and subsequent annual retesting by $100 \%$ agreement on the diagnosis and at least $80 \%$ agreement for symptom presence. JA chaired weekly conference calls to review inclusion criteria for all individuals admitted to the study. DLP trained research assistants in social cognitive assessments. The study protocols and informed consents were reviewed and approved by the ethical review boards of all three sites.

\subsection{Statistical Analyses}

All results were analyzed with IBM SPSS Version 19 and SAS version 9.2. The student ttest and chi-square test were used to compare baseline differences between CHR and HSC groups. Mann-Whitney U tests were used as indicated by significant Levene's test for equality of variances $(\mathrm{p}<.05)$. In regards to aim (a), generalized linear mixed model for repeated measures was used to examine change over time (baseline, 6 months, 12 months, 18 months, and 24 months) for ratings on the three social cognitive indices of ToM, trustworthiness, and approachability and for aim (b), hierarchical regression was used to test whether social cognitive variables explain more variance in conversion than IQ score.

Exploratory analyses were conducted to test whether social cognitive variables explain more variance in conversion than symptoms.

\section{Results}

\subsection{Sample Characteristics}

The majority of the sample was Caucasian and single. Fewer than 50\% in each group had not completed high school and more than $40 \%$ in each group were either in or had completed post- secondary education. There were no significant differences between HSC and CHR groups on any demographic variables or IQ score. The CHR group had significantly higher ratings than the HSC group on baseline total positive symptoms and general symptoms of the SOPS than the HC. There were no significant differences on negative or disorganized symptoms (Table 2).

Converters had significantly higher ratings than non-converters on all baseline SOPS symptom scales (positive, negative, disorganized, and general) (Table 2). There was a statistical trend such that the non-converters tended to have higher IQ scores than converters $(\mathrm{z}=-1.87, \mathrm{df}=176, \mathrm{p}=.061)$.

Group comparisons between dropouts and non-dropouts were conducted to investigate potential relationships with demographic variables, clinical characteristics, and social cognitive performance. Dropouts were defined as individuals that did not return for the 6 month visit. Dropouts had a significantly greater number of males and tended to rate faces as significantly less trustworthy compared to the non-dropout group. There were no significant differences in age, race, IQ, baseline symptoms, approachability ratings, or performance on the Eyes Task (Table 3).

\subsection{Longitudinal changes over time as a function of risk}

Results of the mixed effects modelling demonstrated that there were no differences between the CHR group and the HSC group on ToM performance and trustworthiness judgments at baseline or at any of the follow up assessments. However, there was a significant effect of time such that both groups' ToM performance improved over time ( $F=33.88, \mathrm{df}=1,434$, $\mathrm{p}<001)$. There was a significant difference between the CHR and HSC groups on ratings of approachability over time; a group $\times$ time interaction revealed that CHR approachability ratings tended to increase over time (except month 24) and HSC decreased over time $(F=4.01, \mathrm{p}<05)($ Table 4$)$. 


\subsection{Conversion to psychosis}

Twenty-nine participants in the CHR group (14 male, 15 females) and 5 participants in the HSC group ( 2 males, 3 females) converted to psychosis. Baseline social cognitive data was only available for $25 \mathrm{CHR}$ ( 13 male, 12 female) and $3 \mathrm{HSC}$ ( 1 male, 2 females) participants. Data from all converters is used in subsequent analyses.

Regression was used to predict conversion from baseline ToM performance across both $\mathrm{CHR}$ and HSC groups. The model demonstrated that baseline ToM performance significantly predicted conversion across the whole sample $(F=4.99$, d.f. $=1,230, \mathrm{p}<05)$. Converters $(n=28)$ performed more poorly on ToM than non-converters at baseline. In addition, there was a statistical trend whereby baseline trustworthiness predicted conversion in the CHR sample $(F=2.95$, d.f. $=1,143$, $\mathrm{p}=.088)$. Converters $(\mathrm{n}=24)$ rated faces as higher in trustworthiness than non-converters (Table 5).

As ToM ability is mediated to some degree by general IQ score (Harrington et al. 2005; Janssen et al., 2003), hierarchical regression was used to determine if ToM or trustworthiness significantly predicted conversion beyond IQ score. Predictor variables were entered into the model in the following order: (1) IQ, (2) ToM or trustworthiness. ToM was found to be significantly associated with conversion after the effect of IQ was statistically removed, as indicated by change in $\mathrm{R}$ square $(F=4.13, \mathrm{df}=1,175, \mathrm{p}<05)$. Trustworthiness was not significantly related to conversion after controlling for IQ (Table 5).

Evidence also suggests there is an association between social cognitive performance and psychotic symptoms in both populations at risk for developing psychosis (Eack et al., 2010) and chronic schizophrenia (Hamm et al., 2012; Marjoram et al., 2005; Shean et al., 2005). Analyses were therefore conducted to evaluate the relationship between baseline total symptoms and ToM. ToM was significantly correlated with negative symptoms $(\mathrm{r}(229)=-$. $23, \mathrm{p}<000)$ and disorganized symptoms $(\mathrm{r}(229)=-.26, \mathrm{p}<.000)$ at baseline. The relationship between ToM and general symptoms approached statistical significance $(\mathrm{r}(229)=-.12, \mathrm{p}=$. $07)$. ToM was not significantly associated with positive symptoms $(\mathrm{r}(229)=-.03, \mathrm{p}=.63)$

Following this, an additional hierarchical regression was conducted to determine if ToM significantly predicted conversion beyond total baseline symptoms. Predictor variables were entered into the model in the following order: (1) Total SOPS symptoms at baseline (positive, negative, general, and disorganized), (2) ToM. ToM was not found to be significantly associated with conversion after the effect of total baseline symptoms was removed, as indicated by change in $\mathrm{R} 2(F=1.95, \mathrm{df}=1,228, \mathrm{p}=.16)$ (Table 5).

\section{Discussion}

\subsection{Overview}

The aim of this study was to longitudinally investigate theory of mind and social judgments, and their relationship to later conversion to psychosis in a group of people at high risk of developing psychosis. Two main findings emerged from our study. First, we found that over time, CHR individuals tended to judge faces to be more approachable while HSC participants judged faces as less approachable. Second, baseline ToM performance significantly predicted conversion to schizophrenia beyond IQ. However, the contribution of ToM to conversion became non-significant after controlling for total baseline symptoms. These findings will be discussed below. 


\subsection{Longitudinal changes over time as a function of risk}

On average, CHR individuals judged faces to be more approachable over time than HSCs. This finding for increasing approachability ratings over time may seem counterintuitive, as CHR status is often associated with paranoia. However, social judgments demand higherlevel social cognitive processes through the interpretation of more nuanced, subtle social cues. It is suggested patients with schizophrenia tend to rely less on normative social cues when making judgments, resulting in a positive bias (Haut and MacDonald, 2010). Our finding in CHR individuals is consistent with prior research indicating a positive bias in patients with nonparanoid schizophrenia (Couture et al., 2010) and in first-degree family members (Baas et al., 2008b).

There were no significant differences between CHR and HSC groups on ratings of trustworthiness over time. Similar to approachability judgments, there seems to be a trend whereby trustworthiness judgments are becoming more positive over time in the CHR group until month 12 (see Table 4). However, the HSC group showed a similar pattern, thus the interaction was not significant. This calls for further exploration of social decision-making through its possible relationship to symptoms, conversion, and implications for social competence and functioning.

\subsection{Conversion to psychosis}

The participants that converted to psychosis performed significantly worse at baseline than non-converters in Eyes Task performance. This relationship held when controlling for IQ, suggesting that ToM contributes independent variance beyond IQ in predicting conversion to psychosis. This relationship did not hold when controlling for baseline symptoms. Supplemental analyses indicated ToM was significantly correlated with total baseline disorganization and negative symptoms. This is inconsistent with previous findings using the Eyes Task, which indicate ToM is not significantly related to symptoms (Couture et al., 2008; Stanford et al., 2011). However, some work suggests that higher-order ToM is significantly associated with negative and positive symptoms in individuals with prodromal risk syndrome (Green et al., 2012). A shared etiology between ToM and negative and disorganized symptoms has been suggested (Fett et al., 2013). Further, it has been theorized that social cognition and negative symptoms may have some conceptual overlap (Green et al., 2008). It is possible that early social cognitive deficits could be obscured or overshadowed by symptoms and functional decline. This is consistent with theoretical models of positive symptom development, which posit delusions begin with distortions in the perception of social information (Bentall et al., 1994). This calls for a better understanding of the relationship between SC and symptoms in both chronic schizophrenia and CHR groups.

The present study adds to a growing body of evidence exploring relationships between symptoms, social cognition, and neurocognition in predicting transition to psychosis in CHR individuals (Review: Niendam et al., 2009). Future prediction models of psychosis will likely incorporate multiple sources of information, including family history of psychosis, neurocognitive profile, and environmental factors (Review: Fusar-Poli et al., 2013)

\subsection{Limitations}

There are some limitations to the present study. First, the follow-up sample is small because several subjects were lost over time. This created difficulty in looking at social cognitive performance longitudinally, particularly regarding trustworthiness ratings, as dropouts rated faces significantly less trustworthy than non-dropouts. High dropout rates certainly limit conclusions that can be drawn concerning the stability of social cognitive performance in CHR samples. However, most studies of CHR individuals have similar difficulties in 
retaining participants. Given high drop out rates, there may be potential converters that were not captured in the data. Further, as participants converted to schizophrenia, they were not included in subsequent assessments, thus data concerning stability of deficits over time is unavailable.

Additionally, the HSC is not a traditional healthy comparison group. The help-seeking control group is primarily comprised of family members of psychotic individuals and stable attenuated symptom populations; groups that evidence social cognitive deficits (Addington et al., 2012; Janssen et al., 2003). Comparing HSC with CHR individuals may provide a more rigorous test of differences in social cognitive performance, although the HSC group's low-level symptoms deficits may be obscuring potential group differences. Difficulties in theory of mind and social judgments may be evident in individuals that report psychotic-like experiences regardless of whether full criteria are met for a prodromal syndrome.

Lastly, it must be acknowledged that current SC tasks often have significant conceptual and measurement-related overlap (Green et al., 2008; Pinkham et al., 2013). The Eyes task prompts subjects to label pictures of eyes with a word that best categorizes their interpretation of the person's experience (Baron-Cohen et al., 1997). This task is meant to assess ToM, but also likely involves aspects of emotion perception as well. Similarly, the trustworthiness task involves aspects of emotion recognition in ascribing trust or approachability ratings. Thus, it is difficult to make conclusions about specific SC domains and associations with conversion, IQ, and symptomatology.

\subsection{Implications}

The results and limitations lead to recommendations for future research. The present study indicates a need for further work investigating relationships between social cognition, neurocognition, and clinical state in individuals at high clinical risk for developing psychosis. This warrants further exploration of the vulnerability model, as improvement of underlying vulnerability may in itself help reduce risk for later psychosis and limit later functional disability (Cornblatt et al., 2003). Lastly, further work to investigate how these vulnerability markers can be used as treatment targets is critical to developing early intervention efforts.

\section{Acknowledgments}

The authors thank Chris Wiesen, Ph.D. for his assistance in data processing and analysis.

Role of the funding source

This study was supported by the following NIMH grants U01U01MH06634-02 to J. Addington, U01 MH066069-04 to D. Perkins, U01MH066160 to S.Woods.

\section{References}

Addington J, Piskulic D, Perkins D, Woods S, Liu L, Penn D. Affect recognition in people at clinical high risk of psychosis. Schizophr. Res. 2012; 140(1-3):87-92. [PubMed: 22763425]

Adolphs R, Tranel D, Damasio AR. The human amygdala in social judgment. Nature. 1998; 393(6684):470-474. [PubMed: 9624002]

Baas D, Aleman A, Vink M, Ramsey NF, de Haan EH, Kahn RS. Evidence of altered cortical and amygdala activation during social decision-making in schizophrenia. Neuroimage. 2008a; 40(2): 719-727. [PubMed: 18261933]

Baas D, van't Wout M, Aleman A, Kahn RS. Social judgement in clinically stable patients with schizophrenia and healthy relatives: behavioural evidence of social brain dysfunction. Psychol. Med. 2008b; 38(5):747-754. [PubMed: 17988413] 
Baron-Cohen S, Wheelwright S, Hill J, Raste Y, Plumb I. The "Reading the Mind in the Eyes" Test revised version: a study with normal adults, and adults with Asperger syndrome or high-functioning autism. J. Child Psychol. Psychiatry. 2001; 42(2):241-251. [PubMed: 11280420]

Bentall RP, Kinderman P, Kaney S. The self, attributional processes and abnormal beliefs: towards a model of persecutory delusions. Behav. Res. Ther. 1994; 32(3):331-341. [PubMed: 8192633]

Chung YS, Kang DH, Shin NY, Yoo SY, Kwon JS. Deficit of theory of mind in individuals at ultrahigh-risk for schizophrenia. Schizophr. Res. 2008; 99(1-3):111-118. [PubMed: 18096371]

Cannon TD, Cadenhead K, Cornblatt B, Woods SW, Addington J, Walker E, Seidman LJ, Perkins D, Tsuang M, McGlashan T, Heinssen R. Prediction of psychosis in youth at high clinical risk: a multisite longitudinal study in North America. Arch. Gen. Psychiat. 2008; 65(1):28-37. [PubMed: 18180426]

Cohen, J. Statistical Power Analysis for the Behavioural Sciences. New York: Lawrence Erlbaum Associates; 1988.

Cornblatt BA, Lencz T, Smith CW, Correll CU, Auther AM, Nakayama E. The schizophrenia prodrome revisited: a neurodevelopmental perspective. Schizophr. Bull. 2003; 29(4):633-651. [PubMed: 14989404]

Cornblatt BA, Carrion RE, Addington J, Seidman L, Walker EF, Canndon TD, Cadenhead KS, McGlashan TH, Perkins DO, Tsuang MT, Woods SW, Heinssen R, Lencz T. Risk Factors for Psychosis: Impaired Social and Role Functioning. Schizophr. Bull. 2011; 38(6):1247-1257. [PubMed: 22080497]

Couture SM, Penn DL, Addington J, Woods SW, Perkins DO. Assessment of social judgments and complex mental states in the early phases of psychosis. Schizophr. Res. 2008; 100(1-3):237-241. [PubMed: 18255273]

Couture SM, Penn DL, Losh M, Adolphs R, Hurley R, Piven J. Comparison of social cognitive functioning in schizophrenia and high functioning autism: more convergence than divergence. Psychol. Med. 2010; 40(4):569-579. [PubMed: 19671209]

Eack SM, Mermon DE, Montrose DM, Miewald J, Gur RE, Gur RC, Sweeney JA, Keshavan MS. Social cognition deficits among individuals at familial high risk for schizophrenia. Schizophr. Bull. 2010; 36(6):1081-1088. [PubMed: 19366983]

Fett AK, Maat A. GROUP Investigators. Social cognitive impairments and psychotic symptoms: what is the nature of their association? Schizophr. Bull. 2013; 39(1):77-85. [PubMed: 21697150]

Fett AK, Viechtbauer W, Dominguez MD, Penn DL, van Os J, Krabbendam L. The relationship between neurocognition and social cognition with functional outcomes in schizophrenia: a metaanalysis. Neurosci. Biobehav. Rev. 2011; 35(3):573-588. [PubMed: 20620163]

First, M.; Spitzer, R.; Gibbon, M.; Williams, B.; Williams, J. Structured Clinical Interview for DSMIV Axis I Disorders, Patient Edition ed. New York: New York State Psychiatric Institute; 1995.

Fusar-Poli P, Borgwardt S, Bechdolf A, Addington J, Riecher-Rossler A, Schultze-Lutter F, Keshavan M, Wood S, Ruhrmann S, Seidman LJ, Valmaggia L, Cannon T, Velthorst E, De Haan L, Cornblatt B, Bonoldi I, Birchwood M, McGlashan T, Carpenter W, McGorry P, Klosterkotter J, McGuire P, Yung A. The Psychosis High-Risk State: A Comprehensive State-of-the-Art Review. JAMA Psychiatry. 2013; 70(1):107-120. [PubMed: 23165428]

Green MF, Penn DL, Bentall R, Carpenter WT, Gaebel W, Gur RC, Kring AM, Park S, Silverstein SM, Heinssen R. Social cognition in schizophrenia: an NIMH workshop on definitions, assessment, and research opportunities. Schizophr. Bull. 2008; 34(6):1211-1220. [PubMed: 18184635]

Green MF, Bearden CE, Cannon TD, Fiske AP, Hellemann GS, Horan WP, Kee K, Kern RS, Lee J, Sergi MJ, Subotnik KL, Sugar CA, Ventura J, Yee CM, Nuechterlein KH. Social cognition in schizophrenia, part 1: performance across phase of illness. Schizophr. Bull. 2012; 38(4):854-864. [PubMed: 21345917]

Hamm JA, Renard SB, Fogley RL, Leonhardt BL, Dimaggio G, Buck KD, Lysaker PH. Metacognition and social cognition in schizophrenia: stability and relationship to concurrent and prospective symptom assessments. J. Clin. Psychol. 2012; 68(12):1303-1312. [PubMed: 22886716]

Harrington L, Siegert RJ, McClure J. Theory of mind in schizophrenia: a critical review. Cogn. Neuropsychiatry. 2005; 10(4):249-286. [PubMed: 16571462] 
Haut KM, MacDonald AW 3rd. Persecutory delusions and the perception of trustworthiness in unfamiliar faces in schizophrenia. Psychiatry Res. 2010; 178(3):456-460. [PubMed: 20569994]

Hur JW, Byun MS, Shin NY, Shin YS, Kim SN, Jang JH, Kwon JS. General intellectual functioning as a buffer against theory-of-mind deficits in individuals at ultrahigh risk for psychosis. Schizophr. Res. 2013 Advance online publication.

Janssen I, Krabbendam L, Jolles J, van Os J. Alterations in theory of mind in patients with schizophrenia and non-psychotic relatives. Acta Psychiatr. Scand. 2003; 108(2):110-117. [PubMed: 12823167]

Kim HS, Shin NY, Jang JH, Kim E, Shim G, Park HY, Hong KS, Kwon JS. Social cognition and neurocognition as predictors of conversion to psychosis in individuals at ultra-high risk. Schizophr. Res. 2011; 130(1-3):170-175. [PubMed: 21620681]

Marjoram D, Gardner C, Burns J, Miller P, Lawrie SM, Johnstone EC. Symptomatology and social inference: a theory of mind study of schizophrenia and psychotic affective disorder. Cogn. Neuropsychiatry. 2005; 10(5):347-359. [PubMed: 16571466]

McGlashan, T.; Walsh, B.; Woods, S. The Psychosis-Risk Syndrome: Handbook for Diagnosis and Follow-up. New York: Oxford University Press; 2010.

Niendam TA, Jalbrzikowski M, Bearden CE. Exploring predictors of outcome in the psychosis prodrome: implications for early identification and intervention. Neuropsychol. Rev. 2009; 19(3): 280-293. [PubMed: 19597747]

Pinkham AE, Hopfinger JB, Pelphrey KA, Piven J, Penn DL. Neural bases for impaired social cognition in schizophrenia and autism spectrum disorders. Schizophr. Res. 2008; 99(1-3):164175. [PubMed: 18053686]

Pinkham AE, Penn DL, Green MF, Buck B, Healey K, Harvey PD. The Social Cognition Psychometric Evaluation Study: Results of the Expert Survey and RAND Panel. Schizophr. Bull. 2013 Advance online publication.

Shean G, Murphy A, Meyer J. Social cognition and symptom dimensions. J. Nerv. Ment. Dis. 2005; 193(11):751-755. [PubMed: 16260932]

Sprong M, Schothorst P, Vos E, Hox J, van Engeland H. Theory of mind in schizophrenia: metaanalysis. Br. J. Psychiatry. 2007; 191(5-13)

Stanford AD, Messinger J, Malaspina D, Corcoran CM. Theory of Mind in patients at clinical high risk for psychosis. Schizophr. Res. 2011; 131(1-3):11-17. [PubMed: 21757324]

Thompson AD, Bartholomeusz C, Yung AR. Social cognition deficits and the 'ultra high risk' for psychosis population: a review of literature. Early Interv. Psychiatry. 2011; 5(3):192-202. [PubMed: 21726422]

Thompson A, Papas A, Bartholomeusz C, Allott K, Amminger GP, Nelson B, Wood S, Yung A. Social cognition in clinical"at risk" for psychosis and first episode psychosis populations. Schizophr. Res. 2012; 131(2-3):204-209. [PubMed: 22959742]

Wechsler, D. Wechsler Adult Intelligence Scale. 3rd edition ed. Psychological Corporation; 1997.

Wechsler, D. Wechsler Intelligence Scale for Children. 3rd edition ed. Psychological Corporation; 1991. 
Table 1

Number of subjects at follow-up by group

\begin{tabular}{|r|c|c|c|c|}
\hline & \multicolumn{3}{|c|}{ Assessment (years) } \\
\cline { 2 - 5 } & $\mathbf{0 . 5}$ & $\mathbf{1}$ & $\mathbf{1 . 5}$ & $\mathbf{2}$ \\
\hline Completed & & & & \\
HSC & 48 & 46 & 31 & 24 \\
CHR and HSC & 124 & 95 & 65 & 48 \\
\hline Study ended (drop outs) & & & & \\
CHR & 46 & 71 & 85 & 94 \\
HSC & 23 & 33 & 46 & 57 \\
CHR and HSC & 69 & 104 & 131 & 151 \\
\hline Missed Assessment & & & & \\
CHR & 8 & 7 & 7 & 4 \\
HSC & 10 & 3 & 4 & 1 \\
CHR and HSC & 18 & 10 & 11 & 5 \\
\hline CHR & 15 & 20 & 22 & 25 \\
HSC & 3 & 3 & 3 & 3 \\
Converted and HSC & 18 & 23 & 25 & 28 \\
\hline
\end{tabular}




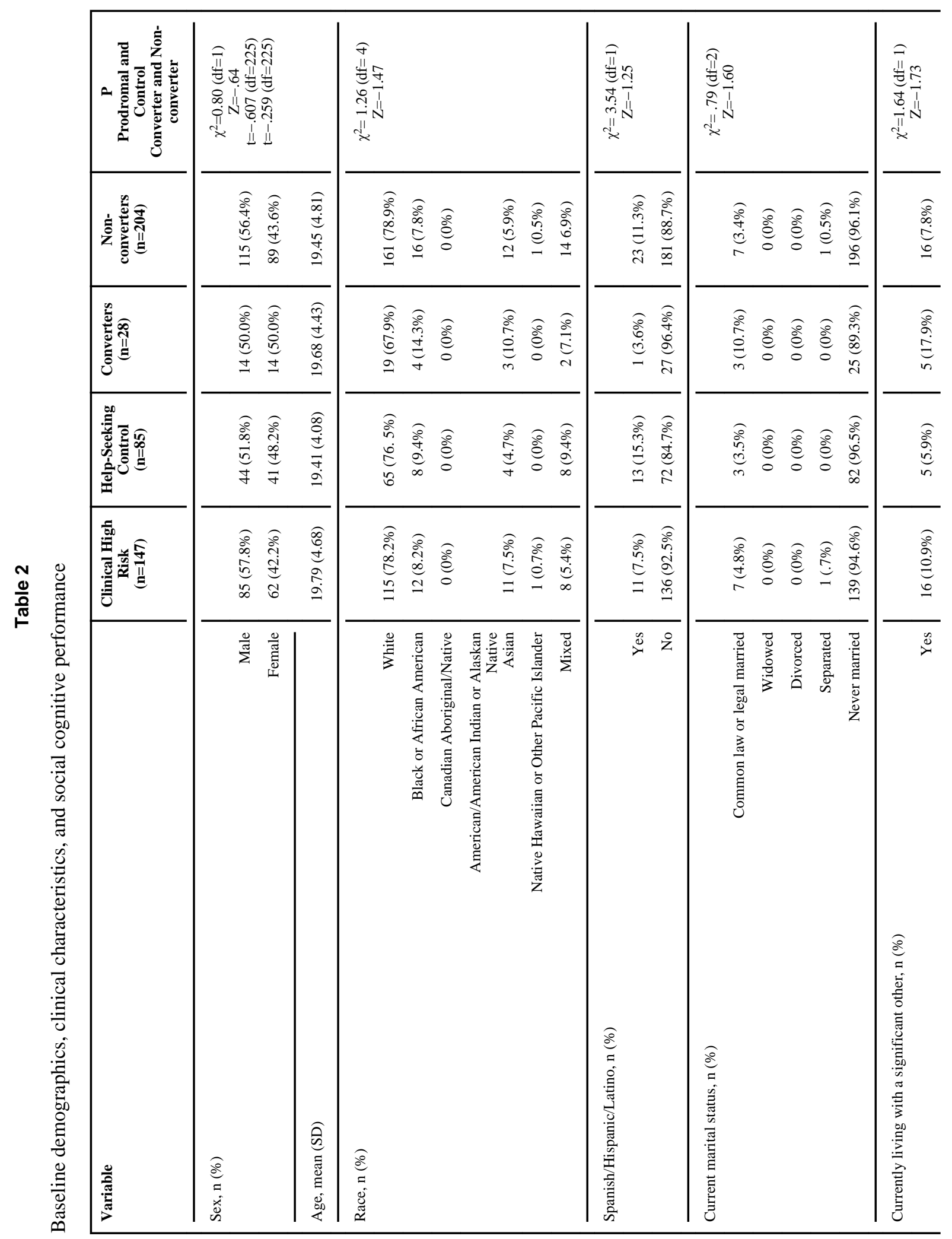

Schizophr Res. Author manuscript; available in PMC 2014 November 01. 


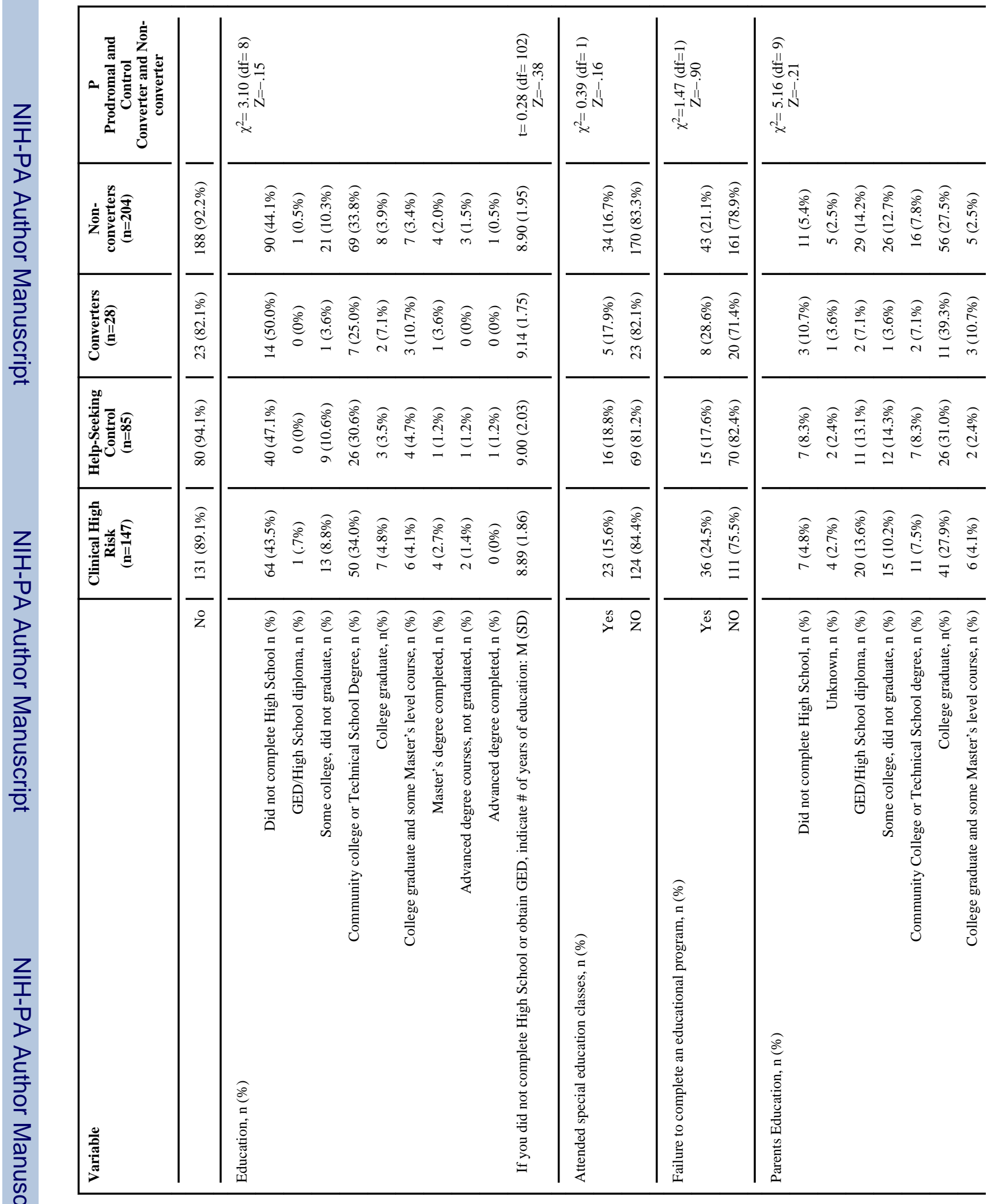




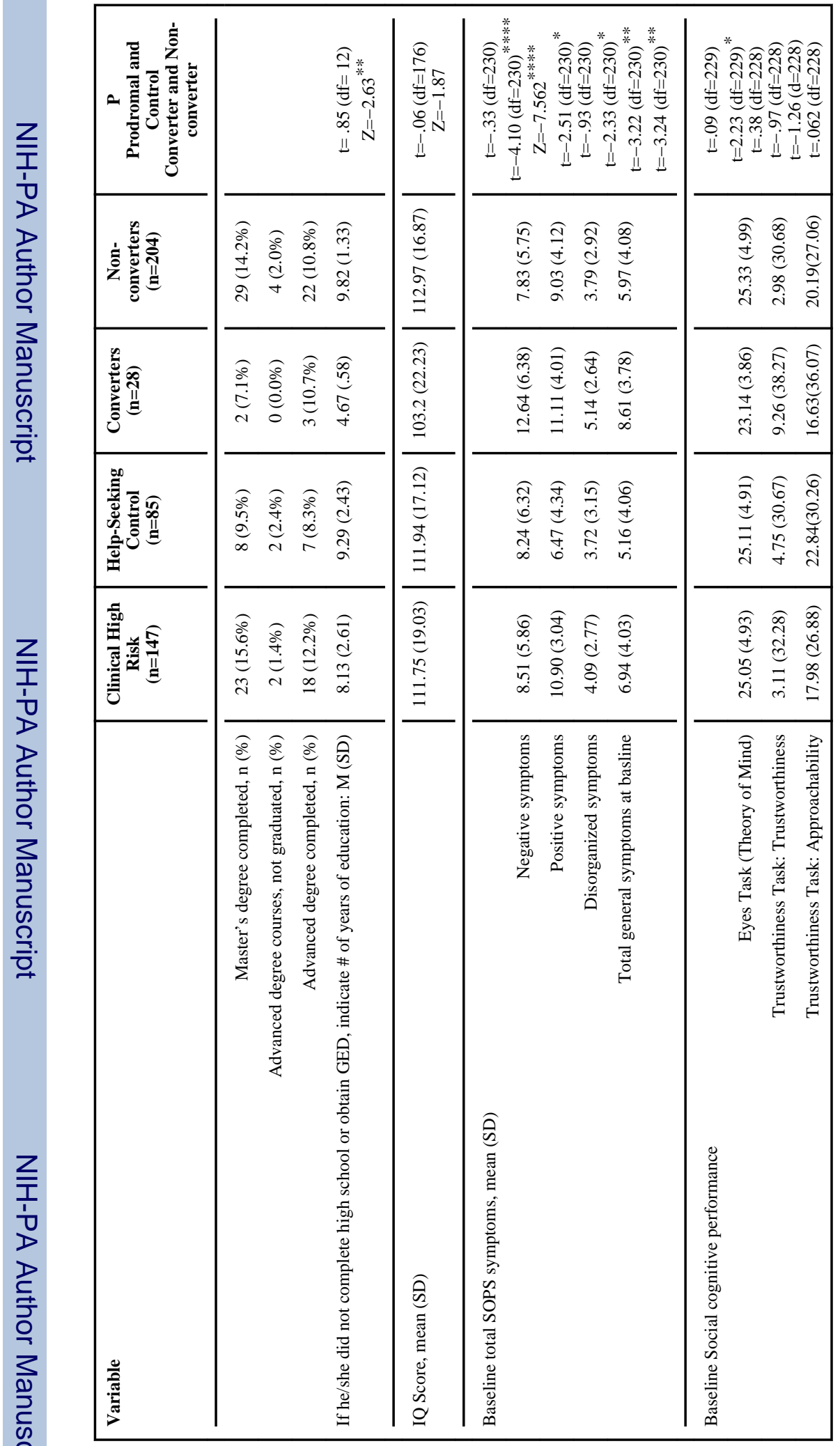

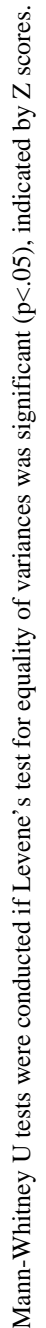

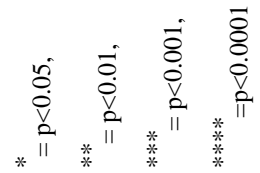




\section{Table 3}

Baseline demographics, clinical characteristics, and social cognitive performance; non-dropouts dropouts.

\begin{tabular}{|c|c|c|c|}
\hline Variable & $\begin{array}{c}\text { Non-dropout } \\
(n=144)\end{array}$ & $\begin{array}{c}\text { Dropout } \\
(n=69)\end{array}$ & $\mathbf{P}$ \\
\hline \multicolumn{4}{|l|}{ Sex, $n(\%)$} \\
\hline Male & $70(48.6 \%)$ & $46(66.7 \%)$ & $\chi^{2}=6.13^{* *}(\mathrm{df}=1)$ \\
\hline Female & $74(51.4 \%)$ & $23(33.3 \%)$ & \\
\hline Age, mean (SD) & $19.44(4.44)$ & $18.57(4.27)$ & $\mathrm{t}=1.359(\mathrm{df}=211)$ \\
\hline Race, $\mathrm{n}(\%)$ & & & $\chi^{2}=1.64(\mathrm{df}=1)$ \\
\hline White & $110(76.4 \%)$ & $58(84.1 \%)$ & \\
\hline Non-white & $34(23.6 \%)$ & $11(15.9 \%)$ & \\
\hline IQ Score, mean (SD) & $113.12(17.38)$ & $110.53(17.49)$ & $\mathrm{t}=.86(\mathrm{df}=165)$ \\
\hline \multicolumn{4}{|l|}{ Baseline total SOPS symptoms mean (SD) } \\
\hline Negative symptoms & $7.90(5.61)$ & $7.94(6.04)$ & $\mathrm{t}=-.06(\mathrm{df}=211)$ \\
\hline Positive symptoms & $9.03(4.22)$ & $9.36(3.90)$ & $\mathrm{t}=-.56(\mathrm{df}=211)$ \\
\hline Disorganized symptoms & $3.67(2.62)$ & $4.09(3.34)$ & $\mathrm{t}=-.98(\mathrm{df}=211)$ \\
\hline Total general symptoms at baseline & $6.27(4.05)$ & $5.58(4.09)$ & $\mathrm{t}=1.16(\mathrm{df}=211)$ \\
\hline \multicolumn{4}{|l|}{ Baseline Social cognitive performance } \\
\hline Eyes Task (Theory of Mind) & $25.38(4.85)$ & $24.78(5.45)$ & $\mathrm{t}=.80(\mathrm{df}=210)$ \\
\hline Trustworthiness Task: Trustworthiness & $7.44(30.57)$ & $-5.29(32.62)$ & $\mathrm{t}=2.78^{* *}(\mathrm{df}=209)$ \\
\hline Trustworthiness Task: Approachability & $21.82(26.60)$ & $15.57(28.31)$ & $\mathrm{t}=1.57(\mathrm{df}=209)$ \\
\hline
\end{tabular}

* $=\mathrm{p}<0.05$,

** $=\mathrm{p}<0.01$,

$* * *$

$=\mathrm{p}<0.001$,

**** $=\mathrm{p}<0.0001$ 


\section{Table 4}

Longitudinal changes over time as a function of risk

\begin{tabular}{|l|c|c|c|c|}
\hline & \multicolumn{2}{|c|}{ Approachability $\boldsymbol{a}$} & \multicolumn{2}{c|}{ Trustworthiness } \\
\hline Month & CHR Mean, SD & HSC Mean, SD & CHR Mean, SD & HSC Mean, SD \\
\hline Baseline & $17.98(26.88)(\mathrm{N}=145)$ & $22.84(30.26)(\mathrm{N}=85)$ & $3.11(32.28)(\mathrm{N}=145)$ & $4.75(30.67)(\mathrm{N}=85)$ \\
\hline Month 6 & $18.62(26.13)(\mathrm{N}=78)$ & $20.38(31.22)(\mathrm{N}=48)$ & $6.12(28.50)(\mathrm{N}=78)$ & $5.15(23.59)(\mathrm{N}=48)$ \\
\hline Month 12 & $20.48(25.48)(\mathrm{N}=50)$ & $20.87(25.24)(\mathrm{N}=32)$ & $12.14(28.65)(\mathrm{N}=50)$ & $6.46(22.54)(\mathrm{N}=46)$ \\
\hline Month 18 & $20.94(31.02)(\mathrm{N}=35)$ & $16.97(23.73)(\mathrm{N}=32)$ & $8.40(28.82)(\mathrm{N}=35)$ & $4.13(25.90)(\mathrm{N}=32)$ \\
\hline Month 24 & $14.58(21.62)(\mathrm{N}=26)$ & $14.42(21.79)(\mathrm{N}=24)$ & $6.54(24.22)(\mathrm{N}=26)$ & $8.63(29.61)(\mathrm{N}=24)$ \\
\hline
\end{tabular}

${ }^{a}$ Significant difference between the CHR and HS group on ratings of approachability over time, with a group by time interaction $(F=4.01, \mathrm{p}<.05)$ 


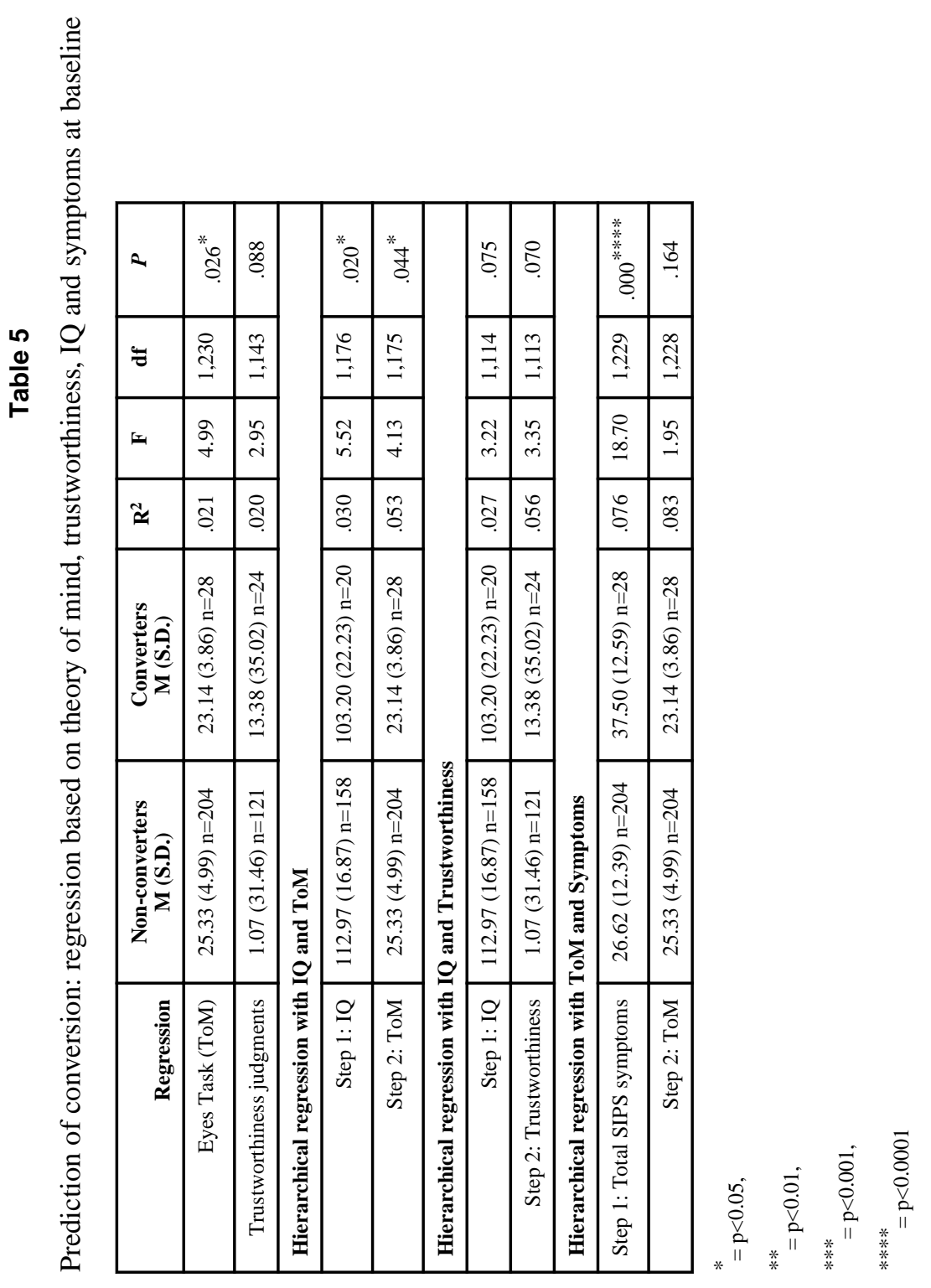

